\title{
EL EFECTO DEL BURNOUT EN LA ANTIGÜEDAD EN EL PUESTO DE TRABAJO: ANÁLISIS MEDIACIONAL DE LA ANSIEDAD
}

\section{THE EFFECT OF BURNOUT IN SENIORITY IN THE WORKPLACE: MEDIATION ANALYSIS OF ANXIETY}

Sagripanti Mazuquin, Omar G., Gonzalez Martín, María J., Messi, Iryna, Romero Illan, Javier, y Khlie Khlie, Youness. Universidad de Granada

Fecha de recepción: 28 de mayo de 2012

Fecha de aceptación: 11 de junio de 2012

Localizador: http:www.ugr.es/local/miguelgr/ReiDoCrea-Vol.1-Art.1-Sagripanti-Gonzalez-Martin-Messi-Romero-Khlie.pdf

\section{Resumen}

Cada vez en mayor medida se hace patente que el síndrome de burnout es una realidad social. Esta disfunción psicológica está relacionada directamente con el ámbito laboral, que afecta especialmente a la profesión sanitaria, caracterizada por una continua relación de ayuda indispensable hacia otras personas, que a su vez dependen del citado personal. La muestra de estudio es de 70 trabajadores de un mismo centro del sector sanitario de atención de mayores de la ciudad de Granada. Los resultados muestran que la ansiedad se relacionará positivamente tanto con el burnout como con la antigüedad en el puesto, mientras que predecimos que a menor realización personal en presencia de mayor antigüedad los valores en ansiedad también serán más altos. También se comprueba que la ansiedad estado tiene efectos mediadores sobre la realización personal, puesto que puede causar mayor alteración de este estado que la propia antigüedad.

Palabras Clave: síndrome de estar quemado, antigüedad, realización personal, sanidad, estrés.

\begin{abstract}
As time goes by it becomes more and more evident that burnout syndrome is a social reality. This psychological dysfunction is directly related to the spheres of labour, especially affecting the sanitary profession, characterized by a continuous relationship of indispensable help towards others, who are dependent on them. The study sample comprised 70 workers of the same healthcare center focusing on elderly care in Granada. The results show that anxiety will correlate positively with burnout as well as with seniority at the job, while we predict anxiety levels will also be higher if there is low personal fulfilment in the presence of more job seniority. It is also proved that a state of anxiety has a mediating effect on personal fulfilment since it can cause a greater alteration in this state than job seniority.
\end{abstract}

Keywords: burnout, seniority, personal fulfilment, health, stress. 


\section{INTRODUCCIÓN}

El estrés laboral es un fenómeno habitual y de interés dadas las consecuencias que conlleva sobre la salud y el bienestar psicológico y físico del individuo (Lazarus y Folkman, 1984). Influye sobre aquellas personas cercanas al afectado y las que reciben un servicio del mismo, al igual que las organizaciones o empresas que requieren de sus servicios. Este deterioro de la eficiencia en el trabajador, fue estudiado en 1974 por el psicólogo estadounidense Freudenberger, que acuñó el término burnout ("estar quemado"), no como un estrés general, sino como síndrome específico de ciertas profesiones que suponen procurar atención a personas en el ámbito de las organizaciones de servicios.

Desde la perspectiva psicosocial, que entiende el burnout como proceso fruto de la interacción de las características del entorno laboral y personales, Maslach y Jackson (1986), conceptualizan el burnout como un síndrome de agotamiento emocional, despersonalización y falta de realización personal en el trabajo que se desarrolla en sujetos cuyo objeto de trabajo son personas. Posteriormente, Maslach y Jackson (1986) utilizaron el mismo término acuñado para describirlo como el proceso de pérdida gradual de responsabilidad y desinterés entre los compañeros de trabajo en el campo de la Psicología Social. Pero la definición más aceptada entre los investigadores es la conceptualizada como el cansancio emocional que lleva a una pérdida de motivación y que suele progresar hacia sentimientos de inadecuación y fracaso (Maslach, Schaufeli y Leiter, 2001). Entre las características que presenta el burnout en médicos se incluye fatiga, desgaste emocional, incapacidad de concentración, ansiedad, insomnio, irritabilidad, incremento del consumo de alcohol o drogas y depresión (Gundersen, 2001).

El síndrome de burnout se compone de tres dimensiones: (a) agotamiento o cansancio emocional definido como el cansancio y fatiga que puede manifestarse física y/o psíquicamente; (b) despersonalización como desarrollo de sentimientos, actitudes y respuestas negativas, distantes y frías hacia otras personas especialmente hacia los beneficiarios del propio trabajo; y (c) baja realización personal o logro que se caracteriza por desilusión para dar sentido a la propia vida y los logros personales con sentimientos de fracaso y baja autoestima (Gil-Monte y Peiró, 1997).

Durante los últimos 20 años, muchos aspectos de la práctica han cambiado y el entorno sanitario en donde nos desenvolvemos está condicionado por diferentes variables, entre los cuales destacamos: nuevas demandas asistenciales, indefinición de las características del trabajo, ambigüedad del rol profesional, crisis del sistema, no solo a nivel económico, sino también académico y de RRHH (Linzer et al., 2001). Otros aspectos notorios son su presumible reorientación de objetivos, la existencia de altos niveles de absentismo laboral, la baja implicación de los profesionales en las organizaciones, la falta de motivación, una paupérrima conciencia de los gestores sobre el problema y la consecuente falta de soluciones desde la organización, la génesis de nuevos sistemas de gestión o la introducción de elementos de competitividad entre los centros. Toda esta amalgama contextual puede suponer que el profesional sanitario deje de lado las necesidades de las personas que recurren a ellos, produciéndose una fingida y encubierta empatía o incluso la falta de ella por el dolor ajeno, generando un estado de ansiedad. Esta ha sido una de las características definitorias del burnout en el sector sanitario (Gundersen, 2001), que tiene un papel como elemento modulador e interviniente en este síndrome. Una de las causas de que la ansiedad aparezca como elemento interviniente, reside en tener que enfrentarse frecuentemente con el sufrimiento humano y la muerte (Vega, 1994). Esto supone un agravante y genera frustración, puesto que la población objeto de estudio -y el sector sanitario en general- suele desarrollar su trabajo por motivos vocacionales. En definitiva, se genera una espiral de sentimientos opuestos encontrados que 
desemboca en un estado de ansiedad, al cual, si le anexionamos la inestabilidad del momento, puede convertirse en una vorágine de descontrol y fatiga emocional.

Los estudios de las variables sociales que pueden tener incidencia en la aparición del burnout se han centrado, hasta el momento, en las variables demográficas de edad, sexo, estado civil, etc. (Savio, 2008). Otra variable de gran importancia es la antigüedad en el puesto, a la que podemos suponer como agravante en dicho estado (Jenaro-Rios, Flores-Robaina y González-Gil, 2007). Ésta junto con la situación contextual que atraviesa el sistema, genera una actitud de alerta que puede generar ansiedad en el trabajador. Esto se ve reflejado en el intento de frustrar al novel, debido, posiblemente, a percibirlo como una amenaza a la estabilidad de sus esquemas de actuación lo que implicaría un cambio de actitud que no están dispuestos a realizar al no haber asumido la nueva filosofía y los modelos que la sustentan, lo que crea una situación amenaza latente que de por sí agrega tensión y ansiedad.

La situación que se plantea no solo se puede expresar en el plano teórico. El burnout, tal como se ha definido en sus tres áreas: agotamiento emocional, despersonalización y falta de realización personal, conducirá al personal sanitario de los centros privados de asistencia sanitaria; principalmente, por su precario estado de crisis institucional en estos momentos; a tener altos índices de ansiedad lo cual repercutirá en mayor burnout generando un bucle de actitudes y conductas propias de dicho cuadro psicológico que también se incrementarán con la antigüedad en dicha institución. Por tanto, partimos de las siguientes hipótesis:

$\mathrm{H1}$ : La ansiedad se relaciona de forma positiva y significativa con las 3 dimensiones del burnout (despersonalización, realización personal y agotamiento emocional).

$\mathrm{H}$ 2: La ansiedad se relaciona de forma positiva y significativa con la antigüedad en el puesto.

H3: La realización personal se relacionará negativamente con la antigüedad en presencia de la ansiedad, es decir, a menor realización personal mayor ansiedad en presencia de una mayor antigüedad.

\section{MÉTODO}

\section{Participantes y procedimiento}

La muestra del estudio estuvo formada por 70 trabajadores de una empresa del sector sanitario, ubicada en la ciudad de Granada. La muestra se encuentra formada en mayor medida por mujeres (90\%). La edad media es de 35.46 años (DT=9,92), mientras que la media de antigüedad en la empresa es de 10,77 años (DT=6,90). Trabajan una media de 42 horas semanales, la mayoría con un contrato indefinido (88 $\%)$.

Los participantes del estudio colaboraron de forma voluntaria y anónima. La devolución del cuestionario se realizó en un sobre cerrado al coordinador del estudio. Los datos se recogieron en el mes de Mayo de 2012. En total de 74 cuestionarios pasados, se recibieron los datos de 70 empleados (tasa de respuesta $=95 \%$ ). 


\section{Instrumentos}

Se construyó un cuestionario que incluía las siguientes medidas:

- Datos sociodemográficos: edad, sexo y años de profesión en el puesto

- Inventario de Ansiedad Estado (State-Trait Anxiety Inventory; STAI) (Spielberger, Gorsuch y Lushene, 2002). En este trabajo se ha utilizado la escala de ansiedad estado de este cuestionario. Esta escala consta de 20 ítems y se responde en una escala tipo Likert de 4 alternativas que oscila entre 0 (Casi nunca) y 3 (Casi siempre). Es una medida con una amplia evidencia acerca de la bondad de sus características psicométricas (Sandín, Valiente, Chorot y Santed, 2005). En el presente trabajo se obtuvo un valor para el coeficiente alfa de Cronbach de 0.94 .

- Cuestionario de frecuencia e intensidad del "burnout" (Maslach Burnout Inventory; MBI), creado por Maslach y Jackson en 1981. Consta de 22 ítems en total y en él se pueden diferenciar tres subescalas, que miden, a su vez los tres factores o dimensiones que conforman el síndrome:

- Agotamiento Emocional (AE): conformado por los ítems 1, 2, 3, 6, 8, 13, 14, 16 y 20. Su puntuación es directamente proporcional a la intensidad del síndrome. Es una medida con amplias características psicométricas que refleja la bondad de la dimensión (Gil-Monte, 2002. En el presente trabajo se obtuvo un coeficiente de fiabilidad alfa de Cronbach de 0,89 para esta dimensión.

- Despersonalización (DP): constituida por los ítems 5, 10, 11, 15 y 22. También su puntuación guarda proporción directa con la intensidad del burnout. En el presente trabajo se obtuvo un coeficiente de fiabilidad alfa de Cronbach de 0,71 para esta dimensión.

- Realización Personal (RP): la conforman los ítems 4, 7, 9, 12, 17, 18, 19 y 21. En este caso la puntuación es inversamente proporcional al grado de burnout. En el presente trabajo se obtuvo un coeficiente de fiabilidad alfa de Cronbach de 0,84 para esta dimensión.

\section{Análisis estadísticos}

Para la realización de los análisis estadísticos se utilizó el paquete estadístico SPSS versión 15.0. En primer lugar se realizaron análisis descriptivos y de correlación para explorar las relaciones entre las variables. Posteriormente se realizaron diversos análisis de mediación. Para ello, se siguieron los pasos recomendados por Baron y Kenny (1986).

\section{RESULTADOS}

\section{Análisis Descriptivos y correlaciones}

Antes de proceder a examinar las hipótesis del estudio se expone una breve reflexión sobre la robustez del constructo "burnout" en la bondad de las correlaciones de sus tres dimensiones, (AE: $\alpha=0,89 ; \mathrm{DP}: \alpha=0,71$; RP: $\alpha=0,84)$, siendo estos coeficientes de fiabilidad lo suficientemente sólidos para considerar la medida de burnout como 
fiable. Es imprescindible mencionar que la correlación intra-dimensional es alta, arrojando puntuaciones contundentes para este estudio (AE-DP: $r=.77$; AE-RP: $r=-$ .65; DP-RP: $r=-.50$ ).

En la tabla 1 se muestran las medias, desviaciones típicas y correlaciones de las variables del estudio. Los datos analizados indican que la ansiedad correlaciona significativa y positivamente de forma elevada con AE $(r=.73 ; p<.01)$, con DP $(r=.56$; $p<, 01)$ y correlaciona negativamente y de forma prominente con RP $(r=-.65 ; p<.01)$. De igual forma la antigüedad correlaciona positivamente con ansiedad $(r=.43 ; p<.01)$ y con RP $(r=-.25 ; p<.05)$.

Tabla 1. Descriptivos y Correlaciones de Pearson entre las distintas dimensiones de Burnout, Ansiedad y Antigüedad en el puesto

\begin{tabular}{lccccccc}
\hline & $\mathrm{M}$ & $\mathrm{DT}$ & Ansiedad & $\mathrm{AE}$ & $\mathrm{DP}$ & $\mathrm{RP}$ & Antigüedad \\
\hline Ansiedad & 38.4 & 13.9 & -- & $.74^{\star \star \star}$ & $.56^{\star \star \star}$ & $-.65^{\star \star \star}$ & $.44^{\star \star \star}$ \\
$\mathrm{AE}$ & 25,9 & 12.6 & & & $.77^{\star \star \star}$ & $-.65^{\star \star \star}$ & .22 \\
$\mathrm{DP}$ & 10.4 & 6.5 & & & & $-.51^{\star \star *}$ & $.23^{*}$ \\
$\mathrm{RP}$ & 27.1 & 8.6 & & & & & $-.25^{\star}$ \\
Antigüedad & 10.7 & 6.9 & & & & & --
\end{tabular}

Nota: AE: Agotamiento Emocional; DP: Despersonalización; RP: Realización Personal

${ }^{* *} p<.001{ }^{* *} p<.01 \quad{ }^{*} p \leq .05$

\section{Análisis de mediación}

Para analizar el posible mediador de la ansiedad estado se siguieron las recomendaciones de Baron y Kenny (1986). Según estos autores, se deben cumplir tres condiciones para mostrar mediación. En primer lugar, que la variable independiente (RP) influya en la variable mediadora (ansiedad). Segundo, que la variable mediadora se relacione con la variable dependiente (antigüedad). Y, en tercer lugar, que exista una relación significativa entre la variable independiente y la variable dependiente, que una vez introducida la variable mediadora pierda, parcial o totalmente, su efecto. Se comprobaron estos requisitos mediante diversos análisis de regresión. Tal y como se aprecia en la tabla 2 , los resultados indican, que la antigüedad en el puesto influye significativamente en la ansiedad $(\beta=.43 ; p<.001)$, de modo que a los participantes con más años en la profesión tenían mayores niveles de ansiedad. A su vez, la antigüedad también predecía la realización personal $(R P)(\beta=-$ $.25 ; p<.05)$. Igualmente, mediante un análisis de regresión independiente, se observó una relación significativa entre ansiedad y $R P(\beta=-.66 ; p<.001)$. Además en el paso 3 de la regresión, se observa que la antigüedad pierde totalmente su efecto sobre la RP $(\beta=.03 ; n . s)$ cuando se introduce la variable ansiedad. Por tanto, de los análisis se desprende que la ansiedad media totalmente la relación entre la antigüedad y la RP (figura 1). 


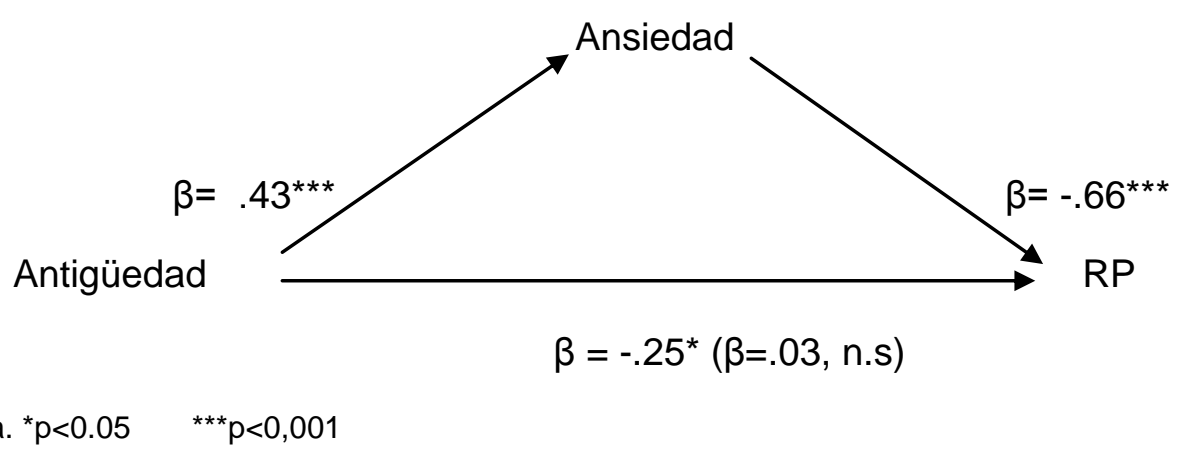

Figura 1. El papel mediador de la Ansiedad en la antigüedad en el puesto y la realización personal

\section{DISCUSIÓN}

El objetivo de este trabajo ha sido desde el principio explorar la relación entre burnout, ansiedad y antigüedad en el puesto. Focalizando el estudio en la población sanitaria que se ve afectada de por sí en su rutina diaria por la estrecha relación de empatía que existe entre el paciente y dicho personal, generando un vínculo afectivo relacional (Camacho Diaz, 2006). Tal concepto es de vital importancia, y discrimina a esta profesión de otras tantas, donde el trabajador no puede dejar de realizar tareas, liberar presiones con "pseudonegligencias", ni reducir o relegar responsabilidades, dado que éste vínculo lo compromete (Naranjo Bermudez y Ricaurte García, 2006). Tal es la importancia de focalizarse en encontrar todas las variables para poder comprender y ayudar a este colectivo. De acuerdo con las hipótesis planteadas, el burnout, la ansiedad y la antigüedad en el puesto generan por separadas un alto estrés, agotamiento emocional, despersonalización, fatiga y disminución de la realización personal, sin embargo si las medimos interactuando, este efecto se potencia (Morales G. y Gallego L. 2004).

Los resultados muestran una relación entre la ansiedad y las dimensiones del burnout, sobre todo el agotamiento emocional, reflejado en la pérdida de energía y la falta de implicación. Asimismo la antigüedad en el puesto, como cabe esperar, tiene una fuerte relación con la ansiedad y a su vez con la realización personal, que en condiciones normales suele afianzar al trabajador a la empresa, pero en la presencia de conflicto puede generar aún mayor ansiedad y frustración.

En la línea de investigaciones previas la edad y la antigüedad en el puesto correlacionan positivamente con las dimensiones del burnout (Jenaro-Río et al., 2007).

Este trabajo a puesto de manifiesto el papel mediador de la ansiedad en la realización personal referida a la antigüedad en el puesto de trabajo, como sabemos cuando este lazo es muy fuerte se produce una atenuación, y en este caso, la casi desaparición de la relación de la antigüedad en el puesto con la realización personal, generando una ansiedad estado mayor y poniendo a los trabajadores en situaciones límites.

Estos resultados justifican la necesidad de intervenir con estos profesionales, dotándoles de estrategias que les permitan hacer frente a las demandas de este tipo 
de trabajos y de realizar mejoras en las propias organizaciones para hacerlas más saludables.

No debemos dejar de aludir a las limitaciones del presente trabajo, el tamaño de la muestra entre ellas, la realización de estudios longitudinales, pre y post evento, ante cualquier eventualidad que surja en la empresa, y por supuesto ampliar la población de trabajo al sistema público de salud.

\section{REFERENCIAS}

Baron R.M, Kenny D.A., (1986). Moderator-mediator variable distinction in social psychological research: Conceptual, strategic, and statistical considerations. Journal of Personality and Social Psychology Journal of Personality and Social Psychology, 51(6), 1173-1182.

Camacho Díaz, J.A. (2006). Aproximación a la empatía en la relación médico-paciente. Bioética \& Debat: Tribuna abierta. Instituto Borja de Bioética, 43, 8-12.

Gil-Monte, P. R. (2002). Validez factorial de la adaptación al español del Maslach Burnout Inventory-General Survey. Salud Publica de México, 1, 3340.

Gil-Monte, P. R. y Peiró, J. (1997). Desgaste psíquico en el trabajo: el síndrome de quemarse. Madrid: Síntesis.

Gundersen, L. (2001). Physician burnout. Annals of Internal Medicine, 135, 145-148.

Jenaro-Río, C., Flores-Robaina, N. y González-Gil, F. (2007). Síndrome de burnout y afrontamiento en trabajadores de acogimiento residencial de menores. International Journal of Clinical and Health Psychology, 7(1), 107-121.

Lazarus R.S. y Folkman, S. (1984). Stress, Appraissal and Coping. New York: Springer.

Linzer, M., Visser, M. R., Oort, F. J., Smets, E. M., McMurray, J. E. y de Haes, H.C. (2001). Predicting and preventing physician burnout: results from the United States and the Netherlands. American Journal of Medicine, 111, 170-175.

Maslach, C. y Jackson, S. (1986). Maslasch Burnout Inventory Manual. Palo Alto California: Consulting Psychology Press.

Maslach, C., Schaufeli, W. B. y Leiter, M. (2001). Job burnout. Annual Review of Psychology, 59, 379-422.

Morales, G., Gallego, L.M. y Rotger, D. (2004). La Incidencia y Relaciones de la Ansiedad y el Burnout en los Profesionales de Intervención en Crisis y Servicios Sociales. 5to congreso virtual de psiquiatría. INTERPSIQUIS.

Naranjo Bermudez, I.C. y Ricaurte García, G.P. (2006). La comunicación con los pacientes. Investigación y Educación en Enfermería, 24(1), 94-98.

Sandín, B., Chorot, P., Santed, M.A., Valiente, R.M. (2005). Propiedades psicométricas del índice de sensibilidad a la ansiedad. Psicothema, 17, 478-483. 
Savio, S. A. (2008). El Síndrome del Burnout: un proceso de estrés laboral crónico. Hologramática, 5(8), 121-138.

Sanz Vergel A.I y Rodríguez Múñoz, A., (2011). El efecto del acoso psicológico en el trabajo sobre la salud: el papel mediador del conflicto trabajo. Revista de Psicología del Trabajo y de las Organizaciones, 27(2), 93-102.

Spielberger, C.D., Gorsuch, R.L., y Lushene, R.E. (2002). STAl: Manual del Cuestionario de Ansiedad Estado-Rasgo. 6ª edición. Madrid: TEA Ediciones.

Vega, D. (1994). Estudio del síndrome del desgaste profesional entre los médicos de un hospital general. Universidad de Salamanca. 